\title{
Mouth Necrotic Lesion
}

National Cancer Institute

\section{Source}

National Cancer Institute. Mouth Necrotic Lesion. NCI Thesaurus. Code C78452.

A necrotic process affecting the tissues of the mouth. 